7-1-2011

\title{
Clinical pharmacology as a foundation for translational science.
}

Scott A. Waldman

Thomas Jefferson University

R J. Hohl

University of lowa

G L. Kearns

Children's Mercy Hospitals, Kansas City

S J. Swan

American Society for Clinical Pharmacology and Therapeutics, Alexandria, Virginia

A Terzic

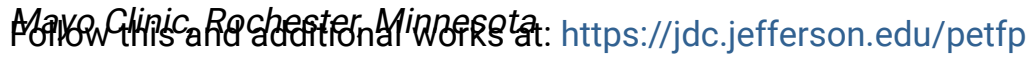

Part of the Medical Pharmacology Commons, and the Pharmacy and Pharmaceutical Sciences Commons

Let us know how access to this document benefits you

\section{Recommended Citation}

Waldman, Scott A.; Hohl, R J.; Kearns, G L.; Swan, S J.; and Terzic, A, "Clinical pharmacology as a foundation for translational science." (2011). Department of Pharmacology and Experimental Therapeutics Faculty Papers. Paper 11.

https://jdc.jefferson.edu/petfp/11

This Article is brought to you for free and open access by the Jefferson Digital Commons. The Jefferson Digital Commons is a service of Thomas Jefferson University's Center for Teaching and Learning (CTL). The Commons is a showcase for Jefferson books and journals, peer-reviewed scholarly publications, unique historical collections from the University archives, and teaching tools. The Jefferson Digital Commons allows researchers and interested readers anywhere in the world to learn about and keep up to date with Jefferson scholarship. This article has been accepted for inclusion in Department of Pharmacology and Experimental Therapeutics Faculty Papers by an authorized administrator of the Jefferson Digital Commons. For more information, please contact: JeffersonDigitalCommons@jefferson.edu. 
As submitted to:

Clinical Pharmacology and Therapeutics

And later published as:

Clinical Pharmacology as a Foundation for Translational

Science

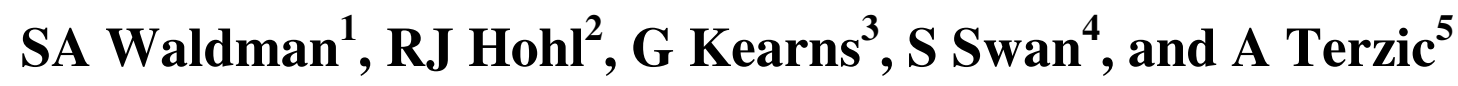

${ }^{1}$ Thomas Jefferson University, Philadelphia, Pennsylvania

${ }^{2}$ University of Iowa, Iowa City, Iowa

${ }^{3}$ The Children's Mercy Hospitals, Kansas City, Missouri

${ }^{4}$ American Society for Clinical Pharmacology and

Therapeutics, Alexandria, Virginia

and

${ }^{5}$ Mayo Clinic, Rochester, Minnesota

(2011) Volume 90 Issue 1, page: 10-13

doi:10.1038/clpt.2011.80

Correspondence

Scott A. Waldman, MD, PhD

Thomas Jefferson University

132 South $10^{\text {th }}$ Street, 1170 Main

Philadelphia, PA 19107

scott.waldman@jefferson.edu

and

Andre Terzic, MD, PhD,

Mayo Clinic

200, First Street SW 
Rochester, MN 55905

terzic.andre@mayo.edu

Title (characters with spaces): $\quad 63$

Word Count in Text Body: 1,374

References:

Display Items: 
The evolution of enabling technologies and their associated perspectives into molecular mechanisms underlying disease has extended beyond the abilities of scientific and clinical structures to advance their translation into new algorithms that improve the health of patients and populations. ${ }^{1}$ Research programs have yielded a vast array of novel molecules related to pathophysiological mechanisms that represent diagnostic and therapeutic targets which have the potential for personalized healthcare management. Yet, despite extraordinary scientific advances, routine successful translation of discovery into new therapeutic tools remains a distant vision. Beyond constraints in bridging discovery science with clinical translation due to obstacles in facilities, resources and in skilled specialized investigators, 95\% of therapies brought into product development by the pharmaceutical and biotechnology sector eventually fail, reflecting negative balance between efficacy and adverse effects.

Appreciating the importance of establishing equilibrium between translation and discovery science, Congress recently enacted the Cures Acceleration Network (CAN), legislation that will create formal bridges between molecular discoveries and new healthcare paradigms. Embracing this mandate to facilitate translation encompassed by CAN, the leadership of the $\mathrm{NIH}$ has created a vigorous path forward by constituting a new National Center for Advancing Translational Sciences (NCATS). The portfolio for this Center includes advancing novel laboratorybased discoveries into potential healthcare technologies with reduced commercial risk, in order to enhance their attractiveness for future development by the private sector. ${ }^{2}$ This mandate will be accomplished by amalgamating programs existing across $\mathrm{NIH}$ that can advance the 
development of scientific discoveries to cross the translational divide. These resources include the Molecular Libraries Program (MLP) which offers high throughput screening capabilities and libraries of compounds useful for research and as therapeutics. For example, the Chemical Genomics Center, part of MLP, provides a robotic, high throughput screening system and a library of $>350,000$ small molecules to interrogate fundamental cellular mechanisms. The Therapeutics for Rare and Neglected Diseases program provides resources for preclinical drug development centered on rare disorders with limited commercial interest. Finally, Clinical and Translational Science Awards offer a nationwide platform of institutions with infrastructures of similar nature and a workforce that ostensibly, could advance the development of therapeutics through patient and community-based studies. Coalescing these resources has tremendous potential to foster interactions between public and private sectors that optimize the utility of technology cores, minimize risk to lower barriers to commercialization, and coordinate transitions across communities of practice (e.g., academic-government, governmentpharmaceutical company) that maximize translational efficiencies.

The genesis of NCATS reflects a modern view of the scientific continuum from molecules to global populations. It envisions the advance of translation from a discipline focused on new therapies that palliate disease, to the quantification of pathobiological risk and disease prevention. ${ }^{3}$ This Center will exploit existing and developing capabilities to optimize the evolution of better patient-centered healthcare algorithms. Further, NCATS will catalyze collaborations between stakeholders emanating from siloed communities within and between discovery, development, and practice, investing them in a public-private partnership 
that is science-based, driving clinical development that enhances efficiencies and reduces risks associated with the development of the next generation of healthcare solutions.

Importantly, NCATS will contribute to producing the next generation of clinical and translational investigators. Equilibrating the balance between discovery and translation requires a cohort of multidisciplinary investigators with fluency in the lexicon and paradigms characterizing the laboratory and clinic, to enable integration of these broadly different domains. ${ }^{4}$ Also, re-balancing entails a transition in professional culture that rewards team science. 5 This envisioned future will require the creation of a brand for clinical and translational science that attracts novice investigators into training programs, to produce the interdisciplinary teams which not only maintain the balance between basic and applied science but most appropriately, are capable of translating a discovery into therapeutic interventions with great promise for good.4, 6,7

The creators of NCATS identified clinical pharmacology as one foundational element essential to success along the discovery-application continuum. ${ }^{8}$ This recognition highlights clinical pharmacology as one discipline at the center of the field of clinical and translational science. The key role for clinical pharmacology in this emerging science reflects its historic position as the index discipline bridging the laboratory and bedside (ie., the translators of translational science). Emanating from the most fundamental inquiries into drug actions, clinical pharmacology focuses on two elementary questions: What do drugs do to the body? and What does the body do to drugs? This concentration on drug action and disposition yielded the earliest paradigms of individualized medicine in which the right drug is used in the right patient at the right dose. 1, 9 
Further, advances in the technologies and approaches emanating from the field of pharmacometrics have further positioned clinical pharmacologists as domain experts at each node of the continuum of drug discovery, development, regulation, and utilization. The continued centrality of clinical pharmacology in translational science is evidenced by its leadership in key international programs linking the laboratory and clinic, including the Pharmacogenomics Research Network10, the Food and Drug Administration's Critical Path Initiative11, and the Institute of Medicine's Committee on Qualification of Biomarkers and Surrogate Endpoints in Chronic Disease ${ }^{12}$.

While NCATS offers a unique opportunity to energize and further develop the firmly established discipline of Clinical Pharmacology in the U.S. and many other parts of the world, it is important that these efforts not be used as either a tangential or overt attempt to "re-brand" clinical pharmacology as the emerging field of clinical and translational science.4, 6, 7 Although translation will be closely served by clinical pharmacology, especially across the continuum of drug discovery, development, regulation, and utilization, these disciplines are intersecting as opposed to their being merely co-existent. The core competencies of clinical pharmacology, including pharmacogenomics, pharmacokinetics, pharmacodynamics, pharmacoepidemiology, pharmacovigilence, and pharmacometrics, to name a few, are tools critical to the therapeutic translational paradigm. However, they also are essential beyond the context of clinical and translational science and they will be applied by investigators who self-identify as clinical pharmacologists, but not translational investigators. Conversely, core elements of clinical and translational science go beyond the facets of clinical pharmacology and 
thus, the portfolio of abilities of translational scientists who are not trained in clinical pharmacology, while valuable to the overall effort/goals of translational science, may not be complete as applied to addressing the intricacies and idiosyncracies of the drug therapy paradigm. The inherent risk in re-branding and folding clinical pharmacology into clinical and translational science is the possibility of dilution, attrition or loss of those central concepts, approaches and techniques that uniquely identify clinical pharmacologists as practitioners. The striking contraction of experienced investigators in the field of whole animal physiology and pharmacology, which were over-shadowed by molecular experimentalists, serves as a warning to resist enveloping clinical pharmacology as a discipline, into clinical and translational science, least we lose the key elements of the former, as a neglected step-child to the higher profile elements in clinical and translational science.

The new National Center for Advancing Translational Sciences represents one essential step in advancing the science of translation. It consolidates national resources and programs whose integration will maximize the discovery-translation continuum. It will crystallize partnerships among academic, industry, and government organizations that will accelerate the evolution of new insights in molecular pathobiology into novel diagnostics and therapeutic tools in the armamentarium available for patient care. Further, it will create a professional development path in clinical and translational science and medicine that will prepare the cadre of specialized investigators invested in the culture of team science. This great promise and potential can only be realized in the context of solving the great challenges that work to minimize the impact of translation on the healthcare of populations: inclusion of broad 
communities of practice, failure rates in drug development, metrics of performance, and covering the translational medicine continuum. The goal for the new Center is to gain early adoption of the integration of discovery science into validated transformational patient-centered algorithms to address unmet needs in healthcare. The critical path to realizing this goal leverages the core competencies encompassing clinical pharmacology as one essential and critical discipline forming the foundation for the emerging field of clinical and translational science. The clinical pharmacology community embraces this goal and looks forward to closely collaborating with NCATS, its leaders, and the diverse communities of practice contributing to the discovery-application paradigm to translate this vision into a reality that improves the lives of our patients.

\section{FINANCIAL DISCLOSURES}

The authors have no relevant disclosures. 


\section{REFERENCES}

(1) Waldman, S.A. \& Terzic, A. Molecular therapeutics from knowledge to delivery. Clin Pharmacol Ther 87, 619-23 (2010).

(2) Butler, D. Translational research: crossing the valley of death. Nature $453,840-2(2008)$.

(3) Clancy, C. \& Collins, F.S. Patient-Centered Outcomes Research Institute: the intersection of science and health care. Sci Transl Med $2,37 \mathrm{~cm} 18(2010)$.

(4) Skarke, C. \& FitzGerald, G.A. Training translators for smart drug discovery. Sci Transl Med 2, 26cm 12 (2010).

(5) Waldman, S.A. \& Terzic, A. Translational medicine in the era of health care reform. Clin Transl Sci 2, 96-7 (2009).

(6) Fitzgerald, G. Drug development needs a new brand of science. Nature 468, 869 (2010).

(7) Fitzgerald, G.A. Clinical pharmacology or translational medicine and therapeutics: reinvent or rebrand and expand? Clin Pharmacol Ther 81, 19-20 (2007).

(8) Rubenstein, A. Translational Medicine and Therapeutics Working Group November 10, 2010. (2010). Accessed January 30, 20112011.

(9) Waldman, S.A. \& Terzic, A. Molecular therapy drives patient-centric health care paradigms. Clin Transl Sci 3, 170-1 (2010). 
(10) Giacomini, K.M. et al. The pharmacogenetics research network: from SNP discovery to clinical drug response. Clin Pharmacol Ther $81,328-45$ (2007).

(11) Lesko, L.J. Paving the critical path: how can clinical pharmacology help achieve the vision? Clin Pharmacol Ther 81, 170-7 (2007).

(12) Mack, A., Balogh, E. \& Micheel, C.M. Perspectives on biomarker and surrogate endpoint evaluation. (2010). Accessed January 30, 2011 2011. 